\title{
Endoscopic treatment of tracheoesophageal fistula using the over-the-scope-clip system
}

\author{
Shigenaga Matsui, Hiroshi Kashida, Yutaka Asakuma, Masatoshi Kudo \\ Kindai University Faculty of Medicine, Japan
}

An 84-year-old man with dysphagia was referred to our hospital for examination. The patient's medical history included endoscopic submucosal dissection for superficial esophageal cancer 2 years previously. Gastrointestinal endoscopy revealed an esophageal foreign body, a pressthrough pack (PTP) (Fig. 1A). The PTP was successfully removed endoscopically. After extraction of the PTP, the patient presented with continuous fever and a cough. Gastrointestinal endoscopy and fluoroscopy revealed a tracheoesophageal fistula in the esophagus (Fig. 1B,C). The tracheoesophageal fistula was endoscopically closed with the Over-The-Scope Clip (OTSC) system (Fig. 2A). The patient's symptoms were immediately improved. Gastrointestinal endoscopy after 2 months revealed a scar with complete fistula closure (Fig. 2B).

The management of tracheoesophageal fistulas is associated with high morbidity and mortality and remains an interdisciplinary challenge. For patients with benign tracheoesophageal fistulas, treatment is always initially supportive, followed by definitive surgical correction [1]. The OTSC system is a new technique that is becoming established as a reliable method for the endoscopic closure of fistulas, bleeds, perforations and other gastrointestinal lesions [2]. The major benefits of the OTSC are its speed and ease of

Department of Gastroenterology and Hepatology, Kindai University Faculty of Medicine, Japan

\section{Conflict of Interest: None}

Correspondence to: Shigenaga Matsui, MD, PhD, Department of Gastroenterology and Hepatology, Kindai University Faculty of Medicine, Osaka, Japan, Tel.: +81 72366 0221, Fax: +81 72367 2880, e-mail: ma2i@med.kindai.ac.jp

Received 30 May 2017; accepted 1 June 2017 published online 26 July 2017

DOI: https://doi.org/10.20524/aog.2017.0181
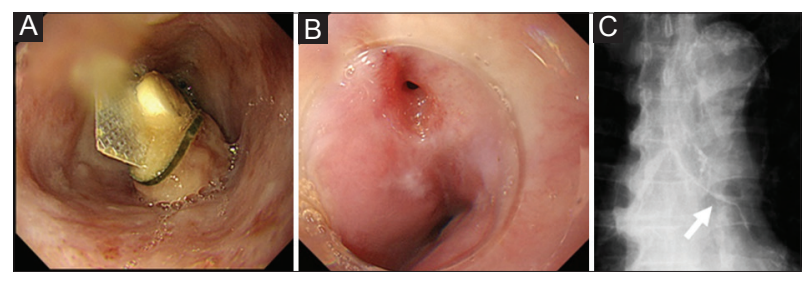

Figure 1 (A) Gastrointestinal endoscopy revealed an esophageal foreign body, a press-through pack. (B) Esophageal orifice of the tracheoesophageal fistula. (C) Fluoroscopy revealed a tracheoesophageal fistula (arrow)

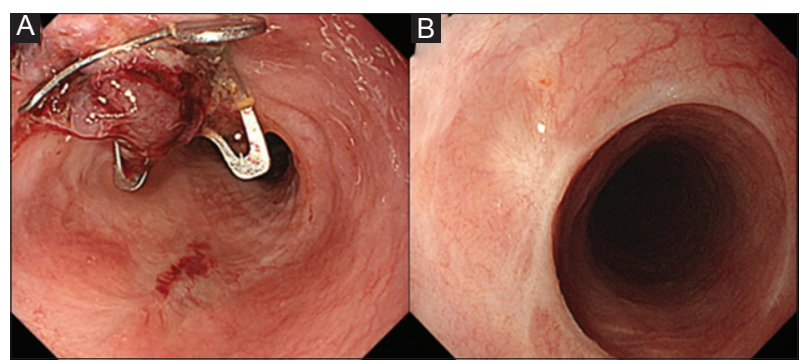

Figure 2 (A) The fistula was closed endoscopically using the over-thescope clip system. (B) Scar of complete fistula closure

deployment, and the persistent sealing of tracheoesophageal fistulas.

\section{References}

1. Reed MF, Mathisen DJ. Tracheoesophageal fistula. Chest Surg Clin N Am 2003;13:271-289.

2. Richter-Schrag HJ, Glatz T, Walker C, Fischer A, Thimme R. Firstline endoscopic treatment with over-the-scope clips significantly improves the primary failure and rebleeding rates in highrisk gastrointestinal bleeding: A single-center experience with 100 cases. World J Gastroenterol 2016;22:9162-9171. 